\title{
Potencial Efeito Antioxidante e Antiproliferativo do Extrato Aquoso de Carya Illinoinensis sobre Células Tumorais
}

\author{
Angelo Viana Weber ${ }^{1}$
}

\begin{abstract}
RESUMO
A oncogênese é um processo fisiopatológico decorrente de um crescimento celular anormal sem que haja ativação de mecanismos que promovam a inibição da proliferação celular. Sendo assim, o tratamento, na maioria dos casos, é baseado na combinação de diferentes técnicas, como, por exemplo, cirurgias, radioterapia e quimioterapia. Os tratamentos por radioterapia e quimioterapia, no entanto, podem gerar a produção de espécies reativas de oxigênio e, consequentemente, o estresse oxidativo. Este estresse oxidativo seria responsável por interferir em muitas funções celulares importantes, principalmente nos locais em que os agentes antineoplásicos exercem sua toxicidade, como na progressão do ciclo celular e apoptose induzida pelos fármacos. Para suprimir o estresse oxidativo, pode-se recomendar a utilização de coadjuvantes no tratamento, como os chás, dentre eles o da casca de noz pecã, Carya illinoinensis. A utilização deste chá, pela crença popular, parece possuir um importante efeito adjuvante no tratamento e prevenção de doenças, como o câncer de colorretal. Para isso, realizou-se uma revisão da literatura nas bases de dados BVS, SciELO e Google Acadêmico. Foram separados 94 artigos e selecionados 21. Os demais artigos foram excluídos por não abordarem a temática pretendida. A Carya illinoinensis apresenta-se como uma fonte natural de antioxidantes, dentre eles os compostos fenólicos e o ácido gálico. Estes compostos estariam demonstrando, em estudos in vitro, atividade antiproliferativa por sua ação citotóxica seletiva, afetando, principalmente, as células tumorais. Muitas propriedades biológicas e farmacológicas de sua composição, todavia, necessitam ser mais bem elucidadas no sentido de compreender os mecanismos de ações envolvidos e, assim, confirmar as suas possíveis ações terapêuticas.
\end{abstract}

Palavras-chave: Carya illinoinensis. Compostos fenólicos. Ácido gálico. Efeito antiproliferativo.

\begin{abstract}
Oncogenesis is a pathophysiological process due to a non-abnormal cell growth that has an activation of mechanisms that promote an inhibition of cell proliferation. Therefore, the treatment in most cases is in the combination of different techniques, such as surgeries, radiotherapy and chemotherapy. However, the treatments by radiotherapy and chemotherapy, can generate a production of reactive species of oxygen, consequently the oxidative stress. This oxidative stress would be responsible for interfering with many important cellular functions, especially where antineoplastic agents exert their toxicity, such as a cell cycle process and drug-induced apoptosis. To suppress oxidative stress, you may recommend using untreated co-adjuvants, such as teas, among them pecan nuts, Carya illinoinensis. The use of this tea by popular belief seems to have a significant adjuvant effect not treating and preventing diseases such as colorectal cancer. To do this, perform a review of the literature in the databases VHL, SciELO and Google Scholar. 94 articles were selected and 21 were selected. The remaining articles were excluded because they did not address an intended theme. Carya illinoinensis presents itself as a natural source of antioxidants, among them phenolic compounds and gallic acid. These compounds would be demonstrating in vitro studies, antiproliferative activity through its selective cytotoxic action, mainly affecting tumor cells. However, many biological and pharmacological properties of its composition need to be better elucidated, there is no sense of pain the mechanisms of actions involved and thus, confirm as their therapeutic actions.
\end{abstract}

Keywords: Carya illinoinensis. Phenolic compounds. Gallic acid. Antiproliferative effect.

Recebido em: 27/1/2017

Revisões requeridas em: 11/4/2017

Aceito em: 25/10/2018 Pós-graduando em Microbiologia Clínica pela Universidade Federal do Rio Grande do Sul - UFRGS, em conjunto com o Instituto de Ciências Básicas da Saúde
- ICBS. Técnico na área de Enfermagem pelo Centro de Educação Básica Francisco de Assis (EFA/Unijuí, 2012). Técnico na área de Citopatologia com ênfase
em citologia cérvico-vaginal (2016) e graduado em Biomedicina com habilitação em Patologia Clínica (2016) pelo Instituto Cenecista de Ensino Superior de
Santo Ângelo (lesa). angelo.vw15@hotmail.com 


\section{INTRODUÇÃO}

O câncer é uma doença em que as células com alterações genéticas crescem de forma anormal, invadindo outros tecidos e perdendo sua função original. As causas primárias ainda não estão muito bem esclarecidas, mas as neoplasias surgem em razão das mutações genéticas espontâneas ou induzidas por agentes patogênicos, como metais, radiações, radicais livres do oxigênio, inflamações crônicas e xenobióticos (tabaco, álcool, pesticidas, etc.), entre outros que promovem desordem no ciclo celular, ocorrendo excesso na taxa de proliferação e deficiência nas taxas de morte celular. Este processo culmina com a formação de agrupamentos de clones de células neoplásicas, isto é, tumores (SANTOS, 2016).

A resposta do organismo humano a este processo é a expressão de alguns marcadores tumorais, que funcionam como indicadores da presença do câncer. Estes marcadores podem ser produzidos diretamente pelo tumor ou pelo organismo em resposta à presença da neoplasia. Além disso, os marcadores tumorais, em sua maioria, são proteínas, antígenos de superfície celular, proteínas citoplasmáticas, enzimas e hormônios (ALMEIDA et al., 2007).

Quando detectados, por meio de exames laboratoriais, os marcadores tumorais comprovam a condição clínica do paciente para o diagnóstico de câncer. O tratamento, na maioria dos casos de câncer, consiste na combinação de diferentes técnicas, como, por exemplo, cirurgias, radioterapia e quimioterapia (BRASIL, 2008). Estudos como de Hubenak et al. (2014) e Galvan (2011), entretanto, mostram que os tratamentos radioterápicos e quimioterápicos podem gerar a produção de espécies reativas de oxigênio e, consequentemente, o estresse oxidativo. Este estresse oxidativo seria responsável por interferir em muitas funções celulares importantes, principalmente nos locais em que os agentes antineoplásicos exercem sua toxicidade, como na progressão do ciclo celular e apoptose induzida pelos fármacos.

Para suprimir o estresse oxidativo que é produzido pelo tratamento, pode-se recomendar a utilização de coadjuvantes de tratamento, como os chás, que possuem efeito antioxidante e antitumoral, como Andrade et al (2011) relatam em sua pesquisa descritiva. O uso do chá, pela crença popular, mostrou-se um importante adjuvante no tratamento e prevenção de doenças, como o câncer de colorretal. Entre as plantas citadas, foi identificado o chá da casca de noz pecã, $C$. illinoinensis, a qual foi caracterizada por possuir ação antioxidante e antitumoral.

\section{METODOLOGIA}

O estudo baseia-se em uma revisão bibliográfica. O processo da revisão foi realizado por meio de uma busca nas bases de dados eletrônicas Scientific Electronic Library Online (Scielo), Biblioteca Virtual em Saúde (BVS) e Google Acadêmico, a partir de artigos publicados em periódicos Nacionais e Internacionais, posteriores ao mês de janeiro de 2000. Os descritores utilizados para a pesquisa foram: Carya illinoinensis, compostos fenólicos e oncogênese. Foram encontrados 893 artigos, os quais passaram por uma análise de título e resumo, para, então, refinar aqueles que estavam mais relacionados ao tema pesquisado. Após essa análise, foram selecionados 94 artigos e realizada uma leitura na íntegra, sendo selecionados 24 artigos. Os demais artigos, num total de 73 , foram excluídos por não abordarem a temática pretendida nesta revisão.

\section{DISCUSSÃO}

Estudos utilizando o extrato da casca de C. illinoinensis em linhagens tumorais, ainda não existem. Pesquisas relacionadas ao mesmo gênero e família da C. illinoinensis, no entanto, são mais frequentemente encontradas. Neste sentido, encontramos a Carya cathayensis e a Juglans regia $\mathrm{L}$. Os estudos com a $C$. cathayensis evidenciaram capacidade antiproliferativa com linhagens tumorais, sendo tal efeito relacionado à ativação de enzimas caspases 3 , que, por sua vez, promoveram uma ação antitumoral (CHEN et al., 2011; LU et al., 2009). Já outros estudos, utilizando a Juglans regia L., demonstraram ação antioxidante e antiproliferativa. Os autores chegam à conclusão que seria pelos compostos fenólicos presentes na noz (SHAH; SHARMA; SHAH, 2015; ANJUM et al., 2016).

Ribeiro (2013) analisou os compostos fenólicos da casca de noz-pecã em extrato aquoso, e reportou concentrações muito baixas de compostos fenólicos, assim como baixa capacidade de ação antioxidante, indo em desacordo com os estudos de Prado et al. (2013), que relataram a presença de altas concentrações de compostos fenólicos e alta capacidade de ação antioxidante, indicando variabilidade nas análises deste extrato. Segundo Gatto (2006), os diferentes níveis de compostos fenólicos devem-se a variações encontradas, como a localização, condições climáticas, variedade da noz, modo de cultivo e maturidade; além disso, a natureza química dessas substâncias fenólicas (como os ácidos fenólicos, antocianinas e taninos), a interação com os outros nutrientes e micronutrientes presentes na amostra e a polaridade do solvente empregado no procedimento da extração. 
A noz pecã apresenta, em sua composição fenólica, cerca de $78 \%$ de ácido gálico, podendo ser encontrados, também, ácidos elágico, $\mathrm{p}$-hidroxibenzoico, protocatequínico, vanílico e quantidades de traços de ácido siríngico (VILLARREAL-LOZOYA, LOMBARDINI, CISNEROS-ZEVALLOS, 2007). Este composto tem demonstrado atividade antiproliferativa por intermédio de sua ação citotóxica seletiva, afetando principalmente células tumorais, tais como leucemia, células cancerosas prostáticas, glioma, câncer gástrico, cólon, peito cervical e esofágico (INOUE et al., 2000; AGARWAL; TYAGI; AGARWAL, 2006; VELURI et al., 2006; KAUR et al., 2009; VERMA; SINGH; MISHRA, 2013; FARIED et al., 2007). Alguns derivados do ácido gálico têm demonstrado efeitos ainda mais potentes que o seu precursor, provavelmente por apresentarem uma maior permeabilidade às membranas plasmáticas.

Russell et al. (2012) avaliaram a atividade do ácido gálico em linhagem de células de câncer de próstata, o qual demonstrou que sua ação antiproliferativa estaria relacionada à indução da apoptose dependente da formação de espécies reativas de oxigênio, as quais seriam produzidas a partir da auto-oxidação do ácido gálico nessa linhagem celular. Essa pesquisa demonstrou que o ácido gálico leva à perda do potencial de membrana mitocondrial, liberação do citocromo $C$ e ativação de caspases 3,8 e 9 . Além disso, Isuzugawa, Inoue e Ogihara (2001) relatam o aumento dos níveis de $\mathrm{Ca}^{2+}$ intracelular, o qual está envolvido na produção de espécies reativas de oxigênio. A modulação do $\mathrm{Ca}^{2+}$ intracelular parece ativar a Caspase 3, que altera a permeabilidade da membrana mitocondrial, levando à apoptose pela via intrínseca.

Os antioxidantes potencializam os efeitos das drogas antineoplásicas, podendo, assim, diminuir a dose administrada desses medicamentos, sem prejudicar o efeito terapêutico, proporcionando a redução dos efeitos colaterais. Essa associação concomitante parece proteger as células sadias da ação das drogas, principalmente as dos tecidos de rápida proliferação celular (SANTOS; SOUZA, 2001).

A formação dos radicais livres e estresse oxidativo pela quimioterapia antineoplásica, porém, embora indesejada, é necessária, pois faz parte do mecanismo de ação destas drogas. A maioria dos agentes antineoplásicos interfere na síntese de ácido desoxirribonucleico (ADN) e ácido ribonucleico (ARN) de proteínas ou no funcionamento adequado de moléculas pré-formadas. Este fato proporciona a liberação de várias substâncias tóxicas ao organismo, que afetam células sadias de tecidos de rápida proliferação celular; além disto, estes agentes não são seletivos e, como consequência, afetam também as células normais (SANTOS; SOUZA, 2001).

A ação dos antioxidantes, portanto, por si só, consegue controlar o crescimento tumoral sem produção de toxicidade, porém com menor eficiência do que as drogas antineoplásicas, mas quando administrados juntos, de forma equilibrada, consegue-se o efeito desejado. Estudos a respeito dessa associação mostram a grande importância da manutenção dos níveis desses nutrientes para o paciente oncológico para proporcionar melhoria da qualidade de vida e maior sobrevida, sendo necessária apenas a ingestão recomendada conforme as Recommended Dietary Allowances (RDAs) e a suplementação para os casos de desnutrição (ROHENKOHL; CARNIEL; COLPO, 2011).

Os extratos de plantas e vegetais podem ser investigados com diferentes propósitos terapêuticos, representando uma importante contribuição para a ciência e a saúde. Estudos com a $C$. illinoinensis ainda são iniciais; o mesmo com o seu subproduto, a casca de noz pecã. Desta forma, por se tratar de uma pesquisa relativamente nova neste contexto, muitas propriedades biológicas e farmacológicas de sua composição necessitam ser analisadas mais detalhadamente, trabalhando de forma individual cada composto bioativo presente no extrato da noz pecã, no sentido de compreender os seus mecanismos de ação envolvidos e, assim, confirmar as suas possíveis ações terapêuticas.

\section{CONCLUSÃO}

É provável que a inviabilidade celular e a atividade antiproliferativa sejam dependentes de diferentes tipos de compostos fenólicos. Sugere-se a continuidade da avaliação deste extrato, utilizando uma série de concentrações a fim de identificar os possíveis efeitos antiproliferativos e mecanismos de ação envolvidos dentro do processo da oncogênese. Também se faz necessária a utilização de outras técnicas que envolvam estresse oxidativo e danos genéticos para o melhor conhecimento destes processos celulares.

Além disso, a purificação dos diferentes compostos fenólicos presentes nos extratos da C. illinoinensis também se torna importante na elucidação do mecanismo de ação sobre as células tumorais, para, assim, quantificá-los e avaliá-los quanto a sua eficácia como compostos antioxidantes e antiproliferativos, e, consequentemente, o entendimento da fisiologia destes compostos sobre a linhagem tumoral. 
O conhecimento da fisiologia e da bioquímica do estresse oxidativo na saúde e nas doenças, nos faz compreender que tanto os mecanismos antioxidantes quanto os mecanismos oxidantes são importantes para o bem-estar do organismo. Para pacientes que fazem o tratamento por quimioterapia e radioterapia, no entanto, torna-se necessário uma atenção maior, posto que a terapêutica tem por objetivo a produção deste estresse oxidativo. Desta maneira, faz-se necessário entender estes mecanismos, vinculados ao tratamento, a fim de elucidar melhor se a neutralização dos radicais livres no tecido tumoral seria algo desejável ou indesejável.

\section{REFERÊNCIAS}

AGARWAL, C.; TYAGI, A.; AGARWAL, R. Gallic acid causes inactivating phosphorylation of cdc25A/cdc25C-cdc2 via ATM-Chk2 activation, leading to cell cycle arrest, and induces apoptosis in human prostate carcinoma DU145 cells. Molecular Cancer Therapeutics, v. 5, n. 12, p. 3.294-3.302, 2006.

ALMEIDA, J. R. C. de et al. Marcadores tumorais: revisão de literatura. Revista Brasileira de Cancerologia, v. 53, n. 3, p. 305-316, 2007.

ANDRADE, F. P. et al. Medicinal plants used for cancer survivors in the treatment and prevention of this disease. Journal of Nursing Ufpe, on-line, v. 5, n. 4, p. 944-950, 2011.

ANJUM, S. et al. Antioxidant and antiproliferative activity of walnut extract (Juglans regia L.) processed by different methods and identification of compounds using GC/MS and LC/MS technique. Journal of Food Processing and Preservation, p. 1-9, 2016.

BRASIL. Instituto Nacional de Câncer. 3. ed. rev., atual., ampl. Rio de Janeiro, 2008.

CHEN, M. R. et al. Effects of the green peel of Carya cathayens on cell proliferation and caspase-3 expression in HeLa cells. Journal of Jilin Medical College, v. 32, n. 5, 2011.

FARIED, A. et al. Anticancer effects of gallic acid isolated from Indonesian herbal medicine, Phaleria macrocarpa (Scheff.) Boerl, on human cancer cell lines. International Journal of Oncology, v. 30, n. 3, p. 605-613, 2007.

GALVAN, D. Efeito da quimioterapia sobre o peso corporal e o estresse oxidativo em mulheres com câncer de mama. 2011. Dissertação (Mestrado em Nutrição) - Universidade Federal de Santa Catarina, Florianópolis, 2011.

GATTO, D. A. Características tecnológicas do vergamento das madeiras de Lluehea divaricata, Carya illinoinensis e Plantanus xacerifolia como subsídios para o manejo florestal. 2006. Tese (Doutorado em Engenharia Florestal) - Universidade Federal de Santa Maria, Santa Maria, 2006.

HUBENAK, J. R. et al. Mechanisms of injury to normal tissue after radiotherapy: a review. Plastic Reconstructive Surgery, v. 133, n. 1, p. 49-56, 2014.

INOUE, M. et al. Role of reactive oxygen species in gallic acid-induced apoptosis. Biological and Pharmaceutical Bulletin, v. 23, n. 10, p. 1.153-1.157, 2000.
ISUZUGAWA, K.; INOUE, M.; OGIHARA, Y. Ca2+-Dependent caspase activation by gallic acid derivatives. Biological and Pharmaceutical Bulletin, v. 24, n. 7, p. 844-847, 2001.

KAUR, M. et al. Gallic acid, an active constituent of grape seed extract, exhibits anti-proliferative, proapoptotic and antitumorigenic effects against prostate carcinoma xenograft growth in nude mice. Pharmaceutical Research, v. 26, n. 9, p. 2.133-2.140, 2009.

LU, W. et al. Crude flavonoids from Carya cathayensis sargent inhibited HeLa Cells proliferation through induction of apoptosis and cell cycle arrest. Latin American Journal of Pharmacy, v. 28, n. 4, p. 568-573, 2009.

PRADO, A. C. P. et al. Relationship between antioxidant properties and chemical composition of the oil and the Shell of pecan nuts. Industrial Crops and Products, v. 45, p. 64-73, 2013.

RIBEIRO, P. C. E. Avaliação da qualidade oxidativa de margarinas adicionadas de extratos de casca de noz-pecã [Carya illinoinensis (Wangenh) C. Koch] e de alecrim [Rosmarinus Officinallis]. 2013. Dissertação (Mestrado em Ciência dos Alimentos) - Universidade Federal de Santa Catarina, Florianópolis, 2013.

ROHENKOHL, Caroline Cavali; CARNIEL, Ana Paula; COLPO, Elisângela. Consumo de antioxidantes durante tratamento quimioterápico. Arquivo Brasileiro de Cirurgia Digestiva, v. 24, n. 2, p. 107-112, 2011.

RUSSELL, L. H. et al. Autoxidation of gallic acid induces ROS-dependent death in human prostate cancer LNCaP cells. Anticancer Research, v. 32, n. 5, p. 1.595-1.602, 2012.

SANTOS, J. L. de S. Proteoma comparativo entre células-tronco mesenquimais humanas com cariótipo normal e invertido. 2016. Monografia (Graduação) - Universidade Federal do Rio Grande do Norte, Natal-RN, 2016.

SANTOS, H. S.; SOUZA, W. M. A terapia nutricional com vitaminas antioxidantes e o tratamento quimioterápico oncológico. Revista Brasileira de Cancerologia, v. 47, n. 3, p. 303-308, 2001.

SHAH, T. I.; SHARMA, E.; SHAH, G. A. Anti-proliferative, Cytotoxicity and Anti-oxidant Activity of Juglans regia Extract. American Journal of Cancer Prevention, v. 3, n. 2, p. 45-50, 2015.

VELURI, R. et al. Fractionation of grape seed extract and identification of gallic acid as one of the major active constituents causing growth inhibition and apoptotic death of DU145 human prostate carcinoma cells. Carcinogenesis, v. 27, n. 7, p. 1.445-1.453, 2006.

VERMA, S.; SINGH, A.; MISHRA, A. Gallic acid: molecular rival of cancer. Environmental Toxicology and Pharmacology, v. 35, n. 3, p. 473-485, 2013.

VILLARREAL-LOZOYA, J. E.; LOMBARDINI, L.; CISNEROS-ZEVALLOS, L. Phytochemical constituents and antioxidant capacity of different pecan [Carya illinoinensis (Wangenh.) K. Koch] cultivars. Food Chemistry, v. 102, n. 7, p. 1.241-1.249, 2007. 